\title{
End-to-end channel capacity of MAC-PHY cross-layer multiple-hop MIMO relay system with outdated CSI
}

\author{
Pham Thanh Hiep ${ }^{1 *}$, Nguyen Huy Hoang ${ }^{2}$, Sugimoto Chika ${ }^{1}$ and Kohno Ryuji
}

\begin{abstract}
For high end-to-end channel capacity, the amplify-and-forward scheme multiple-hop multiple-input multiple-output relay system is considered. The distance between each transceiver and the transmit power of each relay are optimized to prevent some relays from being the bottleneck and guarantee high end-to-end channel capacity. According to the proposed transmission environment coefficient, the path loss can be described in a new way, and then the distance of multiple-hop relay system can be optimized more simply than the original one. However, when the system has no control on media access control (MAC) layer, performance of the system deteriorates because of the presence of interference signal. Thus, the specific transmission protocol on the MAC layer for multiple-hop system is proposed to reduce the power of interference signal and to obtain high end-to-end channel capacity. According to the proposed transmission protocol on the MAC layer, the system indicates the trade-off between the end-to-end channel capacity and the delay time. The end-to-end channel capacity of the outdated channel state information system is also analyzed.
\end{abstract}

\section{Introduction}

Multiple-input multiple-output (MIMO) relay systems have been discussed in several literatures [1-3]. Additionally, ergodic capacity of the amplify-and-forward relay network is discussed. The links between the relay transmitters and relay receivers are assumed to be parallel [4] and serial $[5,6]$. The end-to-end channel capacity based on the different number of antennas at the transmitter, the relay, and the receiver also has been evaluated $[5,6]$. However, the number of relays considered there (in $[5,6]$ ) is only one.

When the number of antennas in relay is less than the number of antennas in the transmitter and receiver, the capacity of MIMO relay system is lower than that of the original MIMO system. Moreover, when the number of antennas in relay equals that in the transmitter and receiver or more, the MIMO relay system can provide the same average capacity as an original MIMO system. In

\footnotetext{
*Correspondence: phamthanhhiep@gmail.com

${ }^{1}$ Division of Physics Electrical and Computer Engineering, Graduate School of Engineering, Yokohama National University, Yokohama, Kanagawa Prefecture 240-8501, Japan

Full list of author information is available at the end of the article
}

other words, although the number of antennas in relay is larger than that in transmitter and receiver, the capacity of MIMO relay system cannot exceed the capacity of original MIMO system [7-10].

Therefore, in order to achieve a high channel capacity, the multiple-hop relay system is considered $[11,12]$. However, in these papers, the interference signal is assumed to be absent; the performance based on a transmission protocol that only has two phases is analyzed, the signalto-noise ratio (SNR) at the receiver is assumed to be fixed, and the location as well as the transmit power of each transmitter are not dealt. In the multiple-hop MIMO relay system, when the distance between the source (TX) and the destination (RX) is fixed, the distance between the TX to a relay station (RS), RS to RS, and RS to the RX called the distances between transceivers, is shortened. Consequently, according to the number of relay and the location of the relays, the SNR and the capacity are changed. Hence, to achieve the high end-to-end channel capacity, the location of each relay, meaning the distance between each transceiver, needs to be optimized. We have analyzed the performance of half-duplex multiple-hop relay system with the amplify-and-forward strategy [13] and

\section{Springer}

(c) 2013 Thanh Hiep et al: licensee Springer. This is an Open Access article distributed under the terms of the Creative Commons Attribution License (http://creativecommons.org/licenses/by/2.0), which permits unrestricted use, distribution, and reproduction in any medium, provided the original work is properly cited. 
decode-and-forward strategy [14]. However, the multiplehop relay system has been optimized on the physical layer, and the transmission of each relay is assumed to be controlled accurately. In this paper, the transmission protocol on media access control (MAC) layer is proposed and the multiple-hop relay system is optimized on both physical and MAC layers, the PHY-MAC cross-layer. Additionally, the system is analyzed when the outdated channel state information (CSI) at the receiver is taken into account. The channel capacity in this paper is the ergodic channel capacity.

The rest of the paper is organized as follows. We introduce the channel model of perfect CSI multiple-hop MIMO relay system in Section 2. Section 3 is the analysis of the system that has interference. The specific access control on the MAC layer is described in Section 4. The end-to-end channel capacity of the outdated system is analyzed in Section 5. Finally, Section 6 concludes the paper.

\section{Multiple-hop MIMO relay system}

\subsection{Channel model}

We assume that there are many relay nodes, i.e., mobile phone and personal computer, arranged in a straight line from the base station to the receiver. When the receiver wants to receive the information from the base station, it transmits the request message to the base station via the relay node. After receiving the message from the receiver, the relay node detects the CSI and adds its location information into the message and transfers to the other node. When the base station receives the request message, it optimizes the distance and the transmit power based on each transmission protocol of the MAC layer. According to the location information of each relay node added into the message, the base station decides the optimized relay node and its transmit power for the multiple-hop relay system. Finally, the base station starts transmitting the information to the receiver.

Figure 1 shows $m$ relays that intervened in the MIMO relay system. Let $M, N$, and $K_{i} \quad(i=1, \ldots, m)$ denote the number of the antenna at TX, RX, and $\mathrm{RS}_{i}$, respectively. The distance between each transceiver is denoted by $d_{i}(i=0, \ldots, m)$. The distance between TX and RX is fixed as $d_{\text {total }}$. TX and all of the relays employ the amplifyand-forward strategy. Mathematical notations used in this paper are as follows: $x$ and $X$ are scalar variables; $\mathbf{x}$ and $\mathbf{X}$ are vector and matrix variables, respectively; $(\cdot)^{\mathbf{H}}$ is the conjugate transpose.

For easy description, TX and RX are also denoted as $\mathrm{RS}_{0}$ and $\mathrm{RS}_{m+1}$, respectively. Since path loss is taken into consideration, the channel matrix is a composite matrix and is modeled as follows: $\sqrt{l_{i, i+1}} \mathbf{H}_{i}, i=0, \ldots, m$, of which $\mathbf{H}_{i}$ represents the channel matrix between $\mathrm{RS}_{i}$ and $\mathrm{RS}_{i+1}$,

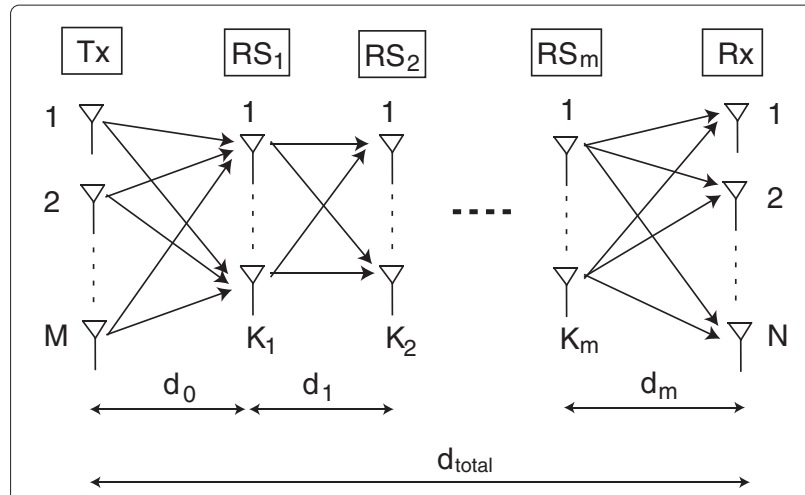

Figure 1 The system model of multiple-hop MIMO relay system.

$l_{i, j}$ denotes the path loss between $\mathrm{RS}_{i}$ and $\mathrm{RS}_{j}$. The path loss is described in detail in the following section. $\mathbf{H}_{i}$ is a matrix with independent and identical distribution (i.i.d), zero mean, unit variance, circularly symmetric complex Gaussian entries.

We assume that the transmit power of $\mathrm{TX}\left(E_{\mathrm{TX}}\right)$ and the total transmit power of relays $\left(E_{\mathrm{RS}}\right)$ are fixed and are not affected by the change in the number of relays and antennas at each relay. In order to simplify the composition of relay and demonstrate the effect of optimizing the distance and the transmit power of each relay, we assume that the transmit power of each relay is equally divided into each antenna, and the number of antennas in each relay is the same. Moreover, zero-forcing algorithm is applied in both the transmitter and the receiver. At first, the system is assumed to be controlled and the interference is absent. All relays transmit the signal at the same time, with full allocation time. Hereafter, this system is called the ideal system, and the end-to-end channel capacity of the ideal system is called the ideal end-toend channel capacity. After that, the interference between every transceiver is taken into consideration, and the specific access control is proposed to obtain high end-to-end channel capacity.

In the ideal system, the ideal end-to-end channel capacity of forward link (from TX to RX) in multiple-hop relay systems is expressed as follows. When the signal $\mathbf{s}_{i-1}$ is transmitted from $\mathrm{RS}_{i-1}$, the received signal at $\mathrm{RS}_{i}$ is expressed as follows:

$$
\mathbf{s}_{i}=\mathbf{H}_{i-i} \beta_{i-1} \mathbf{s}_{i-1}+\mathbf{n}_{i},
$$

where $\mathbf{n}_{i}$ is the noise vector of $\mathrm{RS}_{i}$ and $\beta_{i-1}$ denotes the amplification factor of $\mathrm{RS}_{i-1}$, which is expressed as follows:

$$
\beta_{i-1}=\sqrt{\frac{p_{i-1} l_{i-1, i}}{\left|\mathbf{s}_{i-1}\right|^{2}}},
$$


where $p_{i}$ represents the transmit power of one antenna of $\mathrm{RS}_{i}$.

$$
\begin{aligned}
& E_{\mathrm{TX}}=M p_{0}, \\
& E_{\mathrm{RS}}=\sum_{i=1}^{m} M p_{i} .
\end{aligned}
$$

When $m$ relays are intervened, the signal $\mathbf{s}_{0}$ is transmitted from $\mathrm{TX}$, and the received signal at RX becomes

$$
\mathbf{s}_{m+1}=\sqrt{\frac{p_{m} l_{m, m+1} \ldots p_{0} l_{0,1}}{\left|\mathbf{s}_{m}\right|^{2} \ldots\left|\mathbf{s}_{1}\right|^{2}}} \mathbf{H}_{m} \ldots \mathbf{H}_{1} \mathbf{H}_{0} s_{0}+\mathbf{n}_{\text {system }},
$$

where $\mathbf{n}_{\text {system }}$ denotes the noise vector of the system. Therefore, the system channel matrix is written by

$$
\mathbf{H}=\sqrt{\frac{p_{m} l_{m, m+1} \ldots p_{0} l_{0,1}}{\left|\mathbf{s}_{m}\right|^{2} \ldots\left|\mathbf{s}_{1}\right|^{2}}} \mathbf{H}_{m} \ldots \mathbf{H}_{1} \mathbf{H}_{0}
$$

According to the usage of the system channel matrix $\mathbf{H}$, the multiple-hop MIMO relay system can be analyzed the same as the conventional MIMO system.

\subsection{Ideal end-to-end channel capacity}

Since amplify-and-forward method is applied to the channel model, the noise is also amplified and transmitted to the next relay. Therefore, the system noise vector when $m$ relays are intervened becomes as follows:

$$
\begin{aligned}
\mathbf{n}_{\text {system }}= & \sqrt{\frac{p_{m} l_{m, m+1} \ldots p_{1} l_{1,2}}{\left|\mathbf{s}_{m}\right|^{2} \ldots\left|\mathbf{s}_{1}\right|^{2}}} \mathbf{H}_{m} \ldots \mathbf{H}_{1} \mathbf{n}_{1} \\
& +\sqrt{\frac{p_{m} l_{m, m+1} \ldots p_{2} l_{2,3}}{\left|\mathbf{s}_{m}\right|^{2} \ldots\left|\mathbf{s}_{1}\right|^{2}}} \sqrt{\left|\mathbf{s}_{1}\right|^{2}} \mathbf{H}_{m} \ldots \mathbf{H}_{2} \mathbf{n}_{2} \\
& +\ldots \\
& +\sqrt{\frac{p_{m} l_{m, m+1}}{\left|\mathbf{s}_{m}\right|^{2} \ldots\left|\mathbf{s}_{1}\right|^{2}}} \sqrt{\left|\mathbf{s}_{m-1}\right|^{2} \ldots\left|\mathbf{s}_{1}\right|^{2}} \mathbf{H}_{m} \mathbf{n}_{m} \\
& +\sqrt{\frac{\left|\mathbf{s}_{m}\right|^{2} \ldots\left|\mathbf{s}_{1}\right|^{2}}{\left|\mathbf{s}_{m}\right|^{2} \ldots\left|\mathbf{s}_{1}\right|^{2}}} \mathbf{n}_{m+1} .
\end{aligned}
$$

Since $\mathbf{n}_{i} \mathbf{n}_{i}^{\mathrm{H}}(i=0 \ldots m)$ can be assumed to be a unit matrix, we have

$\mathbf{n}_{\text {system }} \mathbf{n}_{\text {system }}^{\mathrm{H}}=\frac{\sum_{i=0}^{m} \prod_{j \neq i}^{m} l_{j, j+1} p_{j} \sigma^{2}+\sigma^{4} f\left(l_{i, i+1}, p_{j}, \sigma^{2}\right)}{\left|\mathbf{s}_{m}\right|^{2} \ldots\left|\mathbf{s}_{1}\right|^{2}} \mathbf{I}_{M}$,

where $\sigma^{2}$ is the covariance of the noise vector in all relays and $f\left(l_{i, i+1}, p_{j}, \sigma^{2}\right)$ is a polynomial of $l_{i, i+1}, p_{j}, \sigma^{2}$. Since $\sigma^{2} \ll 1$ and the covariance of noise vector are smaller than the received power, the component containing $\sigma^{4}$ can be ignored. Thus, Equation 6 can be changed to the following:

$$
\mathbf{n}_{\text {system }} \mathbf{n}_{\text {system }}^{\mathrm{H}}=\frac{\sum_{i=0}^{m} \prod_{j \neq i}^{m} l_{j, j+1} p_{j} \sigma^{2}}{\left|\mathbf{s}_{m}\right|^{2} \ldots\left|\mathbf{s}_{1}\right|^{2}} \mathbf{I}_{M} .
$$

Consequently, the end-to-end channel capacity of the multiple-hop MIMO relay system is expressed as follows:

$$
\begin{aligned}
C & =\log _{2}\left(\operatorname{det}\left(\frac{\mathbf{s}_{m+1} \mathbf{s}_{m+1}^{\mathrm{H}}}{\mathbf{n}_{\text {system }} \mathbf{n}_{\text {system }}^{\mathrm{H}}}\right)\right) \\
& =\log _{2}\left(\operatorname{det}\left(\mathbf{I}_{M}+\mathbf{H H}^{\mathrm{H}} \frac{\prod_{i=0}^{m} l_{i, i+1} p_{i}}{\sum_{i=0}^{m} \prod_{j \neq i}^{m} l_{j, j+1} p_{j} \sigma^{2}}\right)\right), \\
& =\log _{2}\left(\operatorname{det}\left(\mathbf{I}_{M}+\frac{\mathbf{H H}^{\mathrm{H}}}{\sigma^{2} \sum_{i=0}^{m} \frac{1}{l_{i, i+1} p_{i}}}\right)\right) .
\end{aligned}
$$

Let

$$
f_{m}=\sum_{i=0}^{m} \frac{1}{l_{i, i+1} p_{\mathrm{i}}} .
$$

With the above-mentioned definition of $\mathbf{H}_{i}, \mathbf{H}_{i} \mathbf{H}_{i}^{\mathrm{H}} \quad(i=$ $0, \ldots, m)$ becomes a Gaussian matrix regardless of the number of relays, the distance between each transceiver, and the transmit power of each relay. It means that the end-to-end channel capacity is only restricted by $f_{m}$. Therefore, function $f_{m}$ can be considered instead of the end-to-end channel capacity. In order to achieve the high end-to-end channel capacity, the function $f_{m}$ has to be minimized.

\subsection{Path loss}

As in Equation 8, the system channel matrix is proportional to $p_{\mathrm{i}}$ and $l_{i, i+1}$. Therefore, the path loss plays an important role in the channel model. Since there are a lot of obstacles in the propagation environment, such as huge building, it is necessary to consider the path loss as being attenuated by the reflection. The power of signal is reduced, corresponding to the transmission distance and the number of reflections. An amount of the reduction by one-time reflection is called reflection factor. Naturally, the reflection factor is changed according to the shape of obstacles, the angle of reflections, and so on. However, in this paper, the reflection factor of all reflections is assumed to be the same and denoted by $a$. The path loss is expressed as follows [15-21]:

$$
l_{i, i+1}=\left(\frac{\lambda a^{t_{i}}}{4 \pi d_{i}}\right)^{2}
$$

where $t_{i}$ is the reflected number while the signal is transmitted between $\mathrm{RS}_{i}$ and $\mathrm{RS}_{i+1}$. Additionally, the propagation environment is specified by the propagation environment coefficient. The propagation environment 
coefficient $W$ is defined as the average distance of the line-of-sight distance between each transceiver. Therefore, the reflected number between each transceiver can be expressed as $t_{i}=\frac{d_{i}}{W_{i}}$ and the path loss in Equation 10 can be rewritten in the following new type:

$$
l_{i, i+1}=\left(\frac{\lambda a^{\frac{d_{i}}{W_{i}}}}{4 \pi d_{i}}\right)^{2}
$$

According to the new type, the path loss becomes a function of the distance only. Additionally, the Taylor expression is applied into the term $a^{\frac{d_{i}}{W_{i}}}$, and then the path loss becomes an equation of higher degree of the distance. Therefore, the distance can be optimized easily by the mathematical method or the particle filter method that be explained in the following section.

\section{System that has interference}

\subsection{System model}

Up to now, the interference signal is assumed to be absent and the system has been analyzed. However, when the system has no control on the MAC layer or has incomplete control, the interference signal is present. The received signal at $R S_{i}$ is considered. $\mathrm{RS}_{i}$ simultaneously receives the desired signal $\mathbf{s}_{i-1}$ from $\mathrm{RS}_{i-1}$; the interference signal $\mathbf{s}_{i-2}$, from $\mathrm{RS}_{i-2}$; and the interference signal $\mathbf{s}_{i+1}$, from $\mathrm{RS}_{i+1}$. Actually, $\mathrm{RS}_{i}$ receives the interference signal not only from the neighbor transmitters $\mathrm{RS}_{i-2}$ (forward link) and $\mathrm{RS}_{i+1}$ (backward link), but also from all of the transmitters. However, the interference signal from the other relay is weaker than the interference signal from the neighbor transmitters. Hence, the interference signals from the other relays can be ignored, and the received signal at $\mathrm{RS}_{i}$ is expressed as follows:

$$
\mathbf{s}_{i}=\mathbf{H}_{i-1 i} \mathbf{s}_{i-1}+\mathbf{H}_{i-2 i} \mathbf{s}_{i-2}+\mathbf{H}_{i+1 i} \mathbf{s}_{i+1}+\mathbf{n}_{i},
$$

where $\mathbf{H}_{i j}$ is the channel matrix between $\mathrm{RS}_{j}$ and $\mathrm{RS}_{i}$. Consequently, the end-to-end channel capacity of the system has an interference that can be expressed as follows:

$$
C=\log _{2}\left(\operatorname{det}\left(\mathbf{I}_{M}+\frac{\mathbf{H} \mathbf{H}^{\mathrm{H}}}{f(m)}\right)\right),
$$

where

$$
f(m)=\frac{\sigma^{2}+\sum_{i=1}^{m} l_{i-1, i+1} p_{i-1}+\sum_{i=0}^{m-2} l_{i+1, i+2} p_{i+2}}{\sum_{i=0}^{m} l_{i, i+1} p_{i}} .
$$

In comparison with the ideal end-to-end channel capacity, the term of interference $\sum_{i=1}^{m} l_{i-1, i} p_{i-1}$ of the forward link and $\sum_{i=0}^{m-2} l_{i+1, i+2} p_{i+2}$ of the backward link is added. Similar to the case of the ideal system, the transmit power and the distance can be optimized by mathematical method $[13,14]$. However, the optimization distance of the system with difference $W_{i}$, especially if the system has interference, is complicated. In order to easily optimize the distance and the transmit power simultaneously, the particle filter algorithm is applied.

\subsection{Particle filter method}

In this section, we propose the particle filter (PF) method to optimize both the distance and the transmit powers simultaneously for high end-to-end channel capacity. The algorithm is shown in Figure 2 and explained as follows.

Let $\mathbf{d}$ and $\mathbf{E}$ denote $1 \times m+1$ distance and transmit power vectors, respectively:

$$
\begin{aligned}
& \mathbf{d}=\left[d_{0}, \ldots, d_{m}\right], \\
& \mathbf{E}=\left[E_{\mathrm{TX}}, E_{1}, \ldots, E_{m}\right] .
\end{aligned}
$$

Step 1: $10,000 \times$ random samples of $\mathbf{d}$ and $\mathbf{E}$ are generated. The function $f$ for each sample is calculated, and the minimal value $f$ of all samples is denoted by $\min _{f}$. The sample of $\mathbf{d}$ and $\mathbf{E}$ which has $\min _{f}$ is called the optimal sample.

Step 2: $10,000 \times$ samples of $\mathbf{d}$ and $\mathbf{E}$ are generated around the optimal sample by random function.

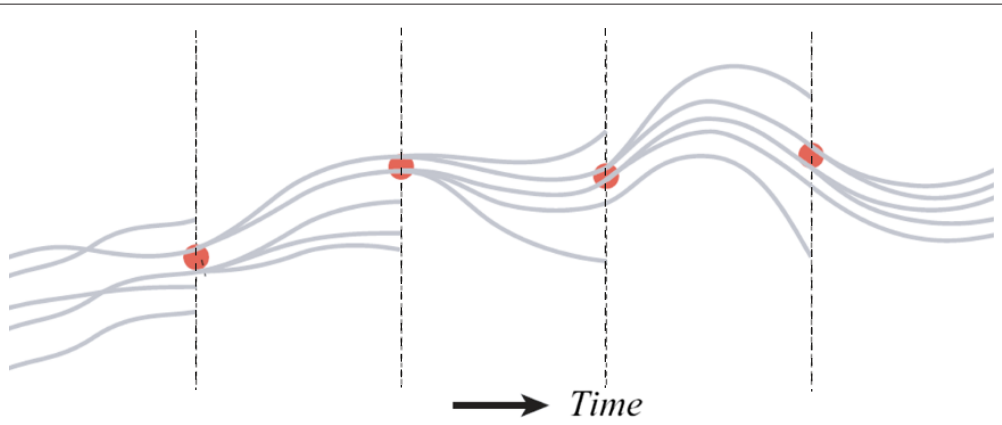

Figure 2 The particle filter algorithm. 
The function $f$ for each sample is calculated and is compared to $\min _{f}$.

Step 3: If there is a function $f$ which is smaller than $\min _{f}$, then $\min _{f}$ is renewed and the process returns to step 2. Otherwise, the algorithm is finished.

To evaluate the particle filter method, the function $f$ in (14) is calculated with the parameters summarized in Table 1. Figure 3 shows the value of function $f$ where the number of relays is 3 and 10 . In the case where the number of relays is 10 , the number of samples is changed, i.e., 1,000 and 10,000. As shown in Figure 3, the value of function $f$ decreases when the number of circles increases, and the algorithm is finished when function $f$ reaches the minimum. The minimum of function $f$ is achieved in the system with average $W$ [14]. Therefore, the algorithm can be finished when function $f$ reaches the value of $f$ in the system with average $W$.

As shown in Figure 3, we can recognize that the number of circles decreases when the number of relays is small and/or the number of samples is larger. However, the PF method requires a large number of samples to converge. If the number of samples is not enough, then the algorithm would not converge or it would take a huge number of circles.

\subsection{Numerical evaluation for the system that has interference}

The system parameter is summarized in Table 1. In order to evaluate the optimization of distance and transmit power. We assume that all relays are arranged in a straight line from the TX to the RX; the transmit power of the RX and the total transmit power of all relays are fixed.

The end-to-end channel capacity of the system in which the transmission of all relays is controlled or not is shown in Figure 4. The end-to-end channel capacity of the system that has interference is much lower than the ideal endto-end channel capacity. Therefore, in order to obtain the high end-to-end channel capacity, the access control on the MAC layer should be considered

Table 1 Numerical parameters

\begin{tabular}{lc}
\hline Antenna elements at TX, RX, RS & $\mathbf{4}$ \\
\hline Transmit power of TX (mW) & 100 \\
Total transmit power of RS (mW) & 100 \\
Noise power $(\mathrm{mW})$ & $6.12 \mathrm{e}-011$ \\
Reflection factor & 0.38 \\
Distance between TX and RX (m) & 3,000 \\
Average LOS W $(\mathrm{m})$ & 500 \\
\hline
\end{tabular}

\section{Specific access control on MAC layer}

\subsection{Multiple-phase transmission}

In this paper, the transmission protocol of all relays is assumed to be controlled on time domain. The transmission of all relays is divided into multiple phases of time domain. The relay in the same phase transmits the signal in the same allocation time that is denoted by $t_{i}$. The other relay that will be divided into different phases keeps the silence or receives the signal. Since the neighbor relay transmits the signal in different phases, the interference signal is weaker than that of the system that has no control. Therefore, the end-to-end channel capacity can be respected to be higher.

Figure 5 shows two and three phases of transmission protocol. The two-phase transmission protocol is explained as follows. The even-number relays and the odd-number relays transmit the signal in phases 1 and 2 , respectively. Therefore, in the two-phase transmission protocol, the closest interference relays of the backward and forward links to $\mathrm{RS}_{i}$ are $\mathrm{RS}_{i+1}$ and $\mathrm{RS}_{i-3}$, respectively.

The end-to-end channel capacity of the system with $n$ phases can be written as follows:

$$
C=\log _{2}\left(\operatorname{det}\left(\mathbf{I}_{M}+\frac{\mathbf{H} \mathbf{H}^{\mathrm{H}}}{f(m)}\right)\right)
$$

where

$f(m)=\frac{\sigma^{2}+\sum_{i=1}^{m+1-n} l_{i-1, i+n} p_{i-1}+\sum_{i=0}^{m-1-n} l_{i, i+1+n} p_{i+1+n}}{\sum_{i=0}^{m} l_{i, i+1} p_{i}}$.

Compare the interference component of the system, it has no control (14) to that of the system with $n$-phase transmission protocol (16), the distance from the interference relay is longer, and the number of interference relay is also larger. Hence, we can say that according to the control on MAC layer, the power of interference decreased; thus, the end-to-end channel capacity is expected to be higher.

\subsection{Numerical evaluation for multiple-phase transmission}

The allocation time for each phase is assumed to be $1 \mathrm{~s}$, and the system model is the same as the one mentioned. In order to evaluate the transmission protocol on the MAC layer, the distance between each transceiver and the transmit power of each relay are assumed to be equal. Figure 6 shows the end-to-end channel capacity in case the access of all relay is controlled on the MAC layer.

For all cases, such as in phases 1 to 4 , we find the optimum number of relays which achieves the highest end-to-end channel capacity. The reason is that, similar to the ideal system, when the number of relay increases, the distance between each transceiver becomes shorter; thus, the power of desired signal increases and the end-toend channel capacity increases. However, the interference 
signal is taken into account. When the number of relays is small, the distance between each transceiver is large; therefore, the power of interference signal is low and the end-to-end channel capacity is high. Moreover, when the number of relays is large, the power of interference signal increases. Therefore, the signal-to-interference noise ratio (SINR) decreases, and the end-to-end channel capacity decreases.

The end-to-end channel capacity of one-phase and twophase transmission protocols is almost the same. The reason is that, compare to two-phase transmission protocol in Subection 4.1, in one-phase transmission protocol, the closest interference relays of backward link and forward link to $\mathrm{RS}_{i}$ are $\mathrm{RS}_{i+1}$ and $\mathrm{RS}_{i-2}$, respectively. Therefore, the power of interference signal to each relay in both phase transmission protocols is almost the same.

The optimum number of relays and the highest end-toend channel capacity are different for each phase. Since the power of interference signal decreases when the number of phases increases, the end-to-end channel capacity of the higher number of phases is higher than that of lower number of phases. In order to evaluate the multiple-hop relay system, the system without relay node, meaning the direct link from the TX to the RX, is taken into consideration. In the case of direct link, the transmit power of $\mathrm{TX}$ is $200 \mathrm{~mW}$ (the sum of transmit power of TX and all relay nodes); the other parameters are the same as those in the multiple-hop relay system (Table 1). The channel capacity of direct link is $6 \times 10^{-4} \mathrm{bit} / \mathrm{s} / \mathrm{Hz}$, which is much smaller than that of the multiple-hop relay system (Figure 6). The reason can be that the signal power is reduced by the distance to the power of 2 and the number of reflections; compared to the direct link, the distance of the multiple-hop relay system is shorter and the number of reflections is smaller. Therefore, although the interference signal is present, the SINR of the multiple-hop relay system is higher than the SNR of the direct link. Since the channel capacity of direct link is much smaller than that of the multiple-hop relay system in the proposed propagation environment, hereafter, only the channel capacity of the multiple-hop relay system is considered. However, the delay time of each scenario should be considered. The delay time is defined as the delay of transmitted signal to the RX compared to the original MIMO system (the direct link). The delay time of the signal transmitted from the TX to the final receiver RX is described in Figure 7. We can recognize that the delay time increases when the number of relays as well as the number of phases increase. It is the trade-off between the end-to-end channel capacity and the delay time.

As shown in Figures 4 and 6, the end-to-end channel capacity of the system that has control on the MAC layer is higher than that of the system that has no control. However, it is smaller than the ideal end-to-end channel capacity. In order to obtain the higher endto-end channel capacity, the distance and the transmit power should be optimized based on each transmission protocol on the MAC layer or the MAC-PHY crosslayer.

\subsection{Numerical evaluation for MAC-PHY cross-layer}

For MAC-PHY cross-layer, for each transmission protocol on the MAC layer, the distance and the transmit power are optimized simultaneously by particle filter method (Subsection 3.2). The allocation time for each phase is assumed to be $1 \mathrm{~s}$, and the system model is the same as mentioned. The end-to-end channel capacity of the MAC-PHY cross-layer is shown in Figure 8.

Similar to the system that only has control on the MAC layer, there is the optimum number of relays which achieves the highest end-to-end channel capacity and the

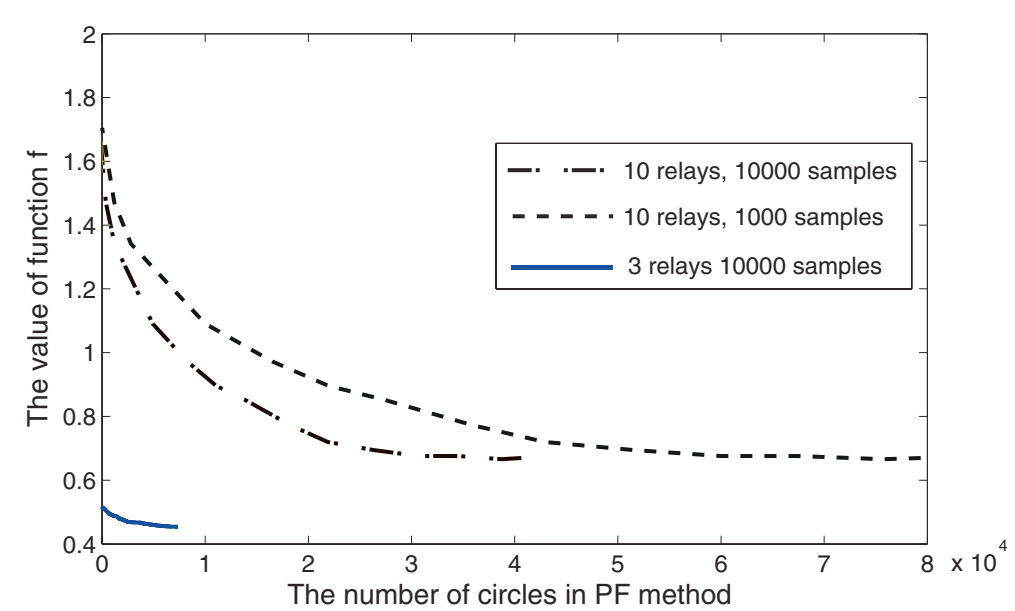

Figure 3 The value of function $f$ that is calculated by particle filter method. 


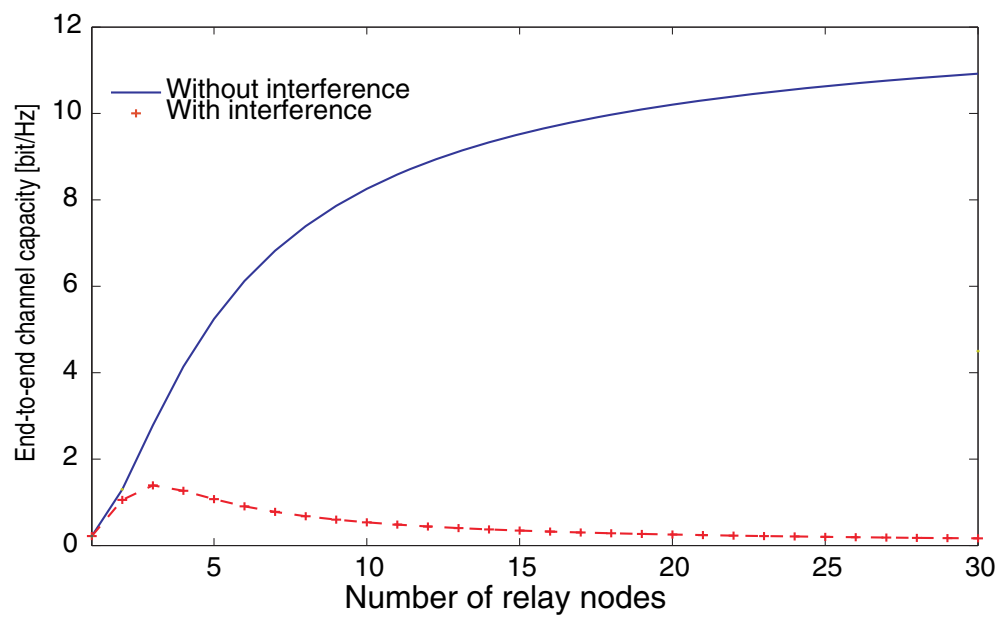

Figure 4 Comparing the end-to-end channel capacity of the ideal system and the system that has interference.

trade-off of channel capacity-delay time. However, comparing to the system that has equal distance and transmit power (Figure 6), the end-to-end channel capacity of the system with MAC-PHY cross-layer is much higher.

Up to now, since the transmission of relay node is assumed to be isotropic, there is the interference from both forward link and backward link. However, the MIMO relay node can use the beamforming technique to transmit the signal to the next relay. It means that the signal is transmitted to the forward link only and that the interference from the backward link is absent. Figure 9 shows the end-to-end channel capacity of system that only has the forward link interference.

As shown in Figure 9, the end-to-end channel capacity increases when the transmission phase increases. Additionally, the trade-off of the channel capacity-delay time can be applied to this scenario. However, comparing to the system that has interference from both forward link and backward link (Figure 8), the end-to-end channel capacity of the system that only has the forward link interference (Figure 9) is much higher. It means that there is the trade-off between complication and channel capacity. The beamforming of MIMO can be applied in the scenario that the higher channel capacity is requested and that the device is large enough to equip the MIMO beamforming.

Up to now, the allocation time of each phase is assumed to be $1 \mathrm{~s}$. Thus, there is the trade-off between the channel capacity and the delay time. However, when the transmission time is normalized, it means that the time to transmit the signal from the TX to the final receiver RX is fixed at $1 \mathrm{~s}$ and the allocation time of each phase is changed, depending on the number of phases. The

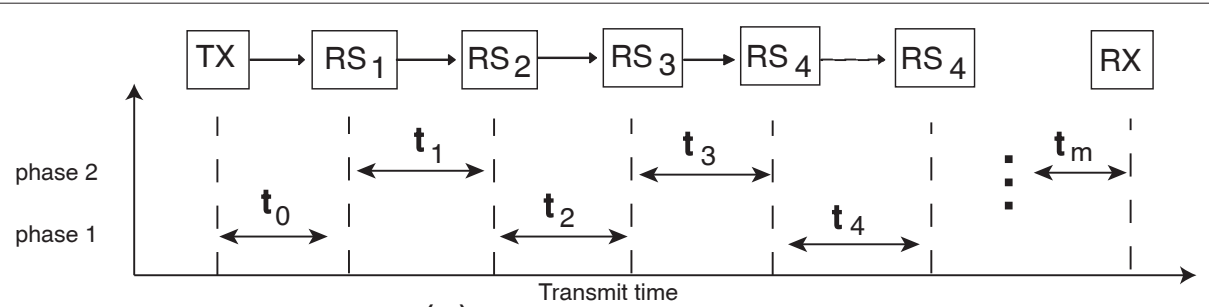

(a) 2 phases transmission

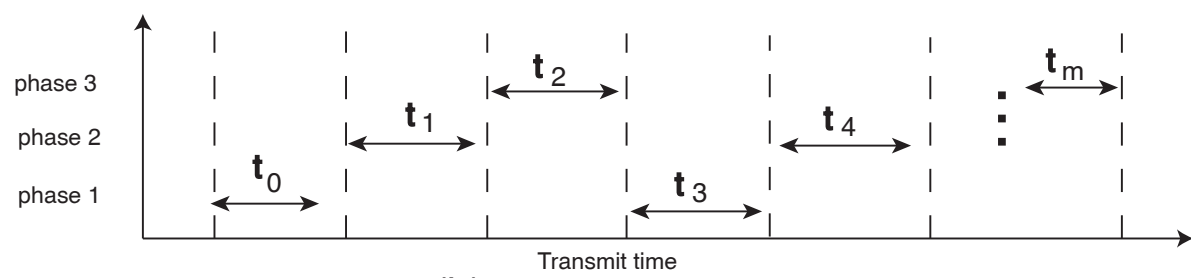

(b) 3 phases transmission

Figure 5 Two-phase (a) and three-phase (b) transmission protocol. 


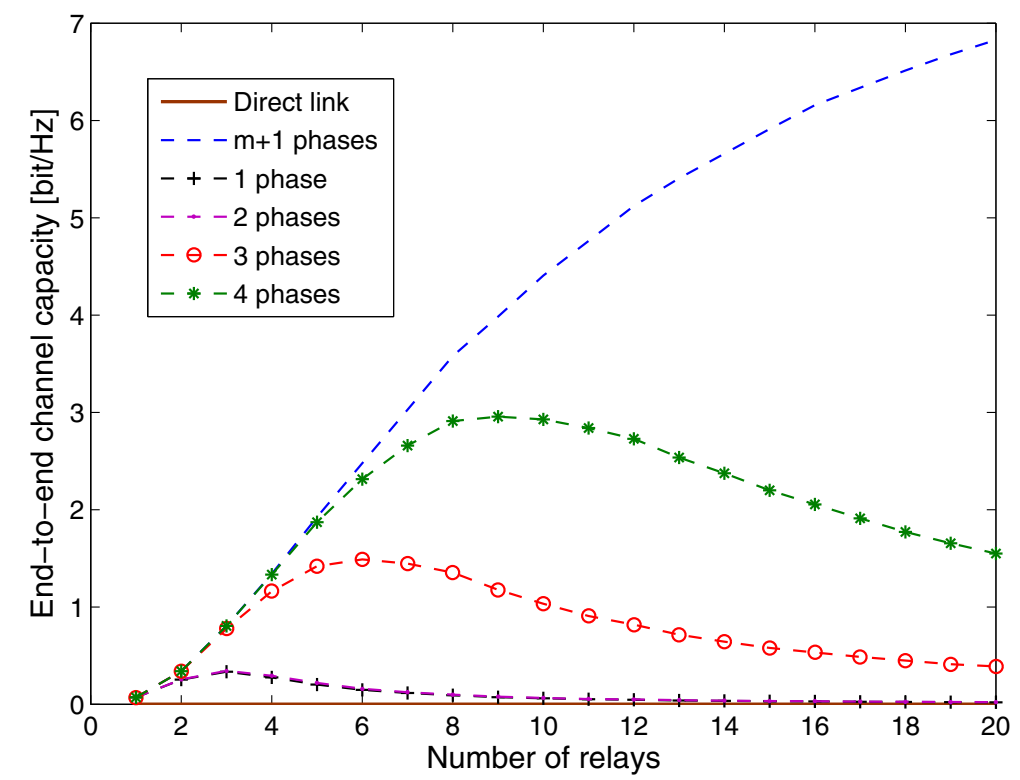

Figure 6 End-to-end channel capacity of the system that has access control on MAC layer. It is compared to the channel capacity of the direct link.

larger the number of phases, the smaller the allocation time of each phase becomes. The system model is the same as mentioned, and the relationship between the end-to-end channel capacity and the number of relays when the transmission time is normalized, as shown in Figure 10. In this system, we also assume that MIMO beamforming is applied for all relays; therefore, only the interference from the forward link is taken into account.
As shown in Figure 10, there are the optimal numbers of relays in the sense of maximal end-to-end channel capacity of each number of phases. Additionally, the maximal end-to-end channel capacity and the optimal number of relays are changed, depending on the transmit power of $\mathrm{TX}$, the total transmit power of RS, the transmission environment $(W)$, and so on. In the case where the number of relays is small, the optimization of the system has no control on the MAC layer (all relays transmit the signal at the

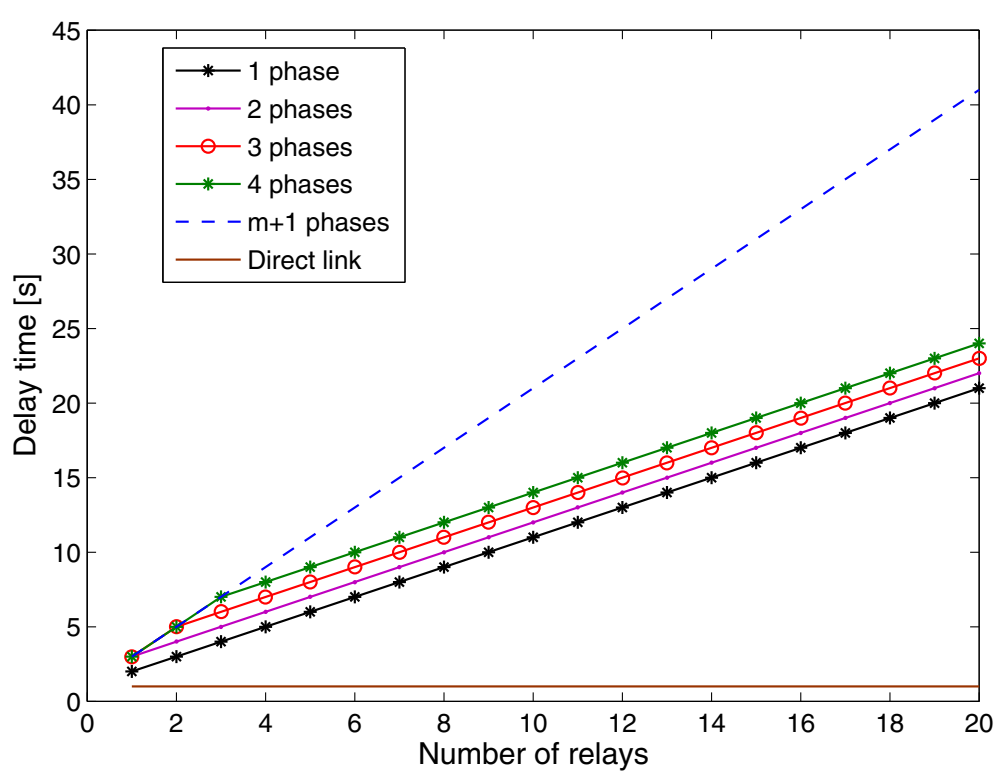

Figure 7 Delay time of multiple-hop relay system for each protocol on the MAC layer and direct link. 


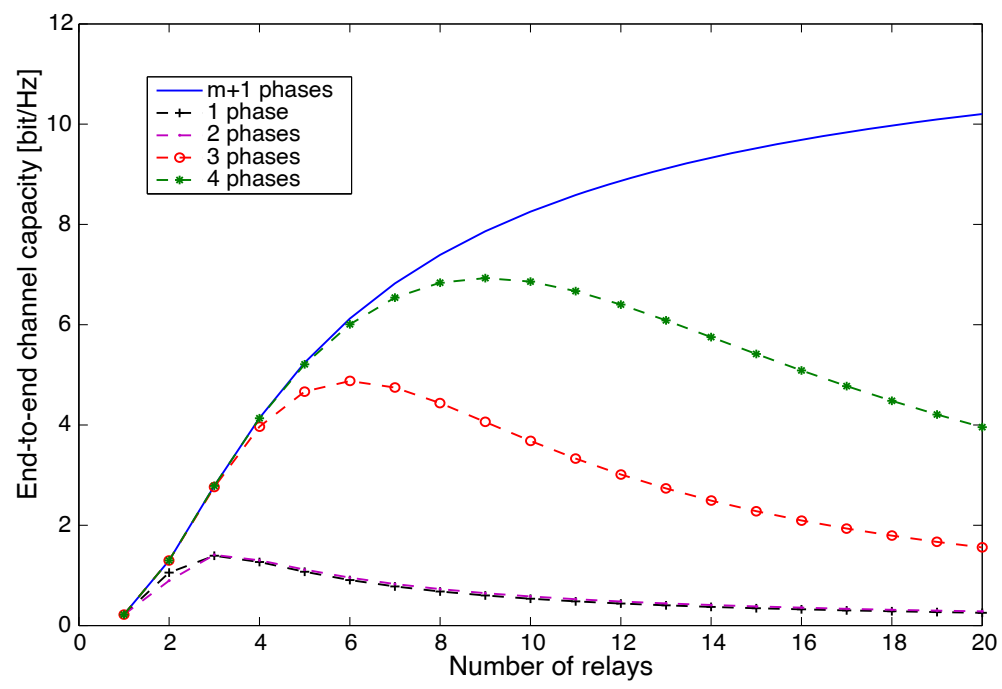

Figure 8 The end-to-end channel capacity of MAC-PHY cross-layer.

same time, one phase) which can reach the higher end-toend channel capacity than in other scenarios. Moreover, in the case where the number of relays is large, the higher number of phases can obtain the higher end-to-end channel capacity. Hence, to obtain the high end-to-end channel capacity, the appropriate number of relays, the number of phases, and so on should be adopted.

\section{Outdated CSI system}

\subsection{System model}

Up to now, the performance of the system is analyzed under the assumption of a perfect CSI at both the transmitter and receiver. However, in actuality, the perfect CSI assumption is not always practical due to channel estimation errors, feedback channel delay, and noise. Compared to channel estimation errors, the CSI imperfection introduced by feedback channel delay is sometimes more significant and inevitable.

To characterize the outdated CSI at the $\mathrm{RS}_{i}$, the channel time variation is described by the first-order Markov process:

$$
\mathbf{H}_{i}(t)=\rho \mathbf{H}_{i}(t-\tau)+k \overline{\mathbf{H}}_{i}(t)
$$

where $\tau$ denotes the total delay caused by signal processing, feedback, and other system delays, $\rho=J_{0}\left(2 \pi f_{D} \tau\right)$ is the time correlation coefficient, $J_{0}(\cdot)$ is the zero-order

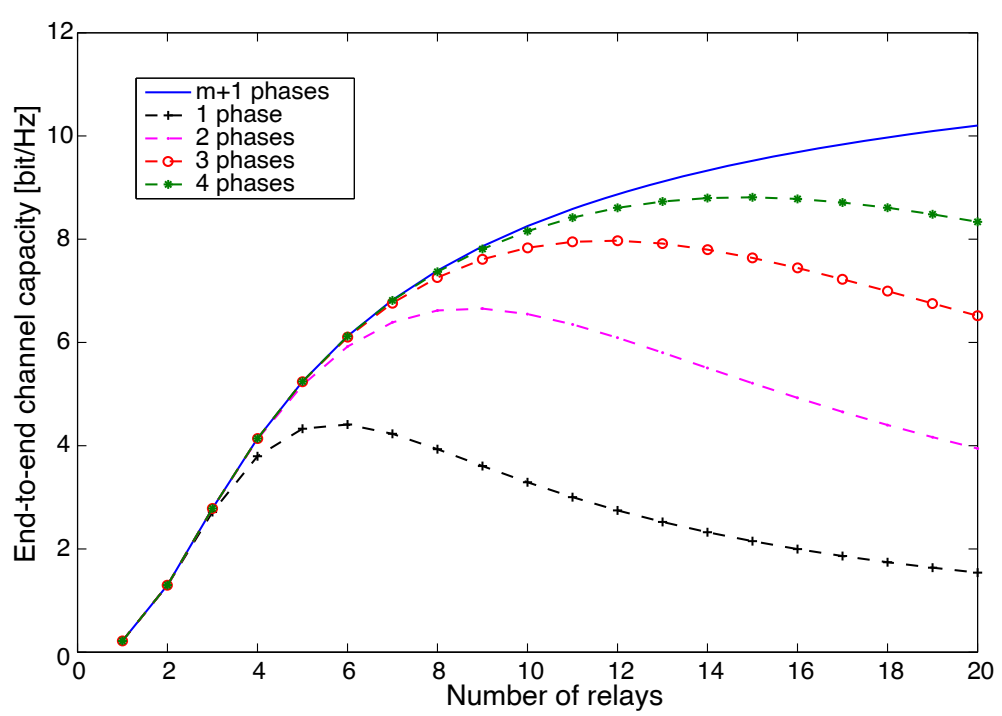

Figure 9 The end-to-end channel capacity of MAC-PHY cross-layer that only has forward link interference. 


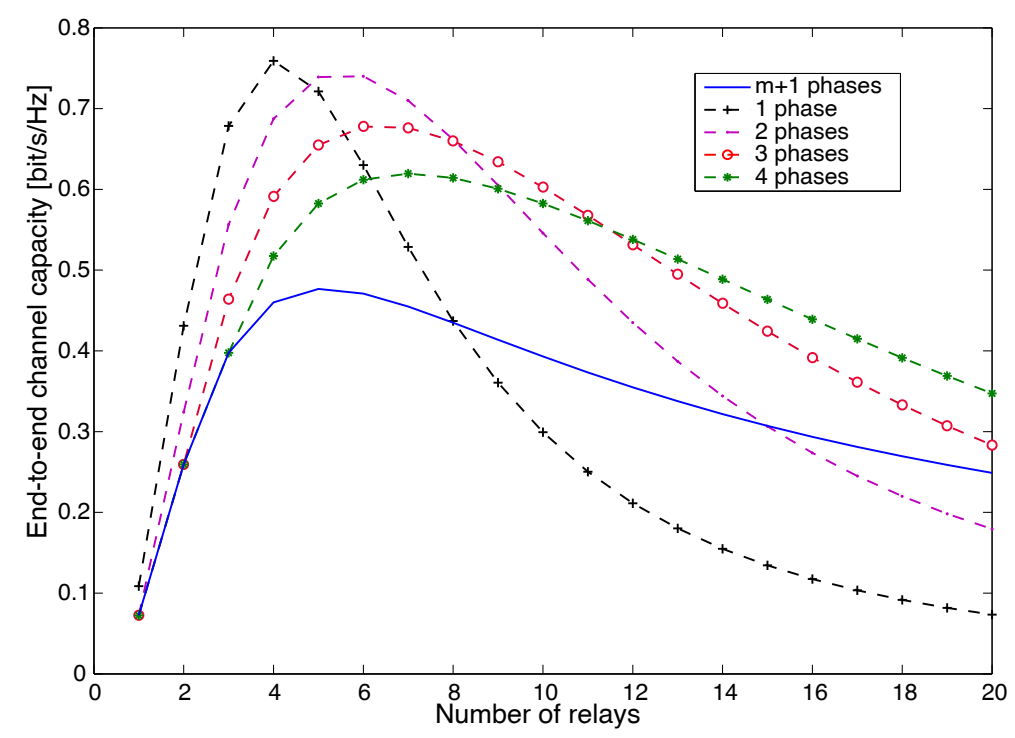

Figure 10 End-to-end channel capacity of the system that has optimization on MAC-PHY cross-layer when transmission time is normalized.

Bessel function of the first kind and $f_{D}$ denotes the maximum Doppler frequency shift. $k$ denotes $\sqrt{1-\rho^{2}}$. The innovation term $\overline{\mathbf{H}}_{i}$ also has i.i.d entries, zero mean, and unit variance. For notation brevity, we drop off the time index:

$$
\mathbf{H}_{i}=\rho \hat{\mathbf{H}}_{i}+k \overline{\mathbf{H}}_{i},
$$

where $\hat{\mathbf{H}}_{i}$ denotes the outdated channel matrix while $\mathbf{H}_{i}$ is the true one.

We assume that the transmitter adds the CSI in the data packet and transmits to the receiver; thus, the receiver knows both $\mathbf{H}_{i}$ and $\hat{\mathbf{H}}_{i}$. After receiving the data packet, the receiver feedbacks the CSI to the transmitter immediately. However, the transmitter should wait some phases to transmit the signal; therefore, this CSI becomes the outdated CSI. It means that the transmitter only knows the outdated CSI $\hat{\mathbf{H}}_{i}$, while the receiver knows both $\mathbf{H}_{i}$ and $\hat{\mathbf{H}}_{i}$. In an SVD-based MIMO system, the relay $\mathrm{RS}_{i}$ steers the modulated signal vector $\mathbf{s}_{i}$ within the eigenspace spanned by the right singular vectors contained in $\hat{\mathbf{E}}_{i}^{t}$ :

$$
\mathbf{s}_{i}^{t}=\hat{\mathbf{E}}_{i}^{t} \mathbf{s}_{i} \text {. }
$$

The relay $\mathrm{RS}_{i+1}$ receives the signal the same way as that in Subsection 2.1:

$$
\mathbf{s}_{i+1}^{r}=\mathbf{H}_{i} \beta_{i} \mathbf{s}_{i}^{t}+\mathbf{n}_{i+1},
$$

and it preprocesses $\mathbf{s}_{i+1}^{r}$ using $\hat{\mathbf{E}}_{i+1}^{r}$ :

$$
\begin{aligned}
\mathbf{s}_{i+1} & =\left(\hat{\mathbf{E}}_{i+1}^{r}\right)^{\mathrm{H}} \mathbf{s}_{i+1}^{r}, \\
& =\left(\hat{\mathbf{E}}_{i+1}^{r}\right)^{\mathrm{H}} \mathbf{H}_{i} \beta_{i} \hat{\mathbf{E}}_{i}^{t} \mathbf{s}_{i}+\left(\hat{\mathbf{E}}_{i+1}^{r}\right)^{\mathrm{H}} \mathbf{n}_{i+1}, \\
& =\rho \hat{\mathbf{D}}_{i+1} \beta_{i} \mathbf{s}_{i}+k\left(\hat{\mathbf{E}}_{i+1}^{r}\right)^{\mathrm{H}} \overline{\mathbf{H}}_{i} \beta_{i} \hat{\mathbf{E}}_{i}^{t} \mathbf{s}_{i}+\left(\hat{\mathbf{E}}_{i+1}^{r}\right)^{\mathrm{H}} \mathbf{n}_{i+1} .
\end{aligned}
$$

We can also use mode complex algorithms such as minimum mean squared errors or maximum likelihood to achieve better performance at the cost of higher complexity. A basic conclusion is that the more complex methods do perform better in outdated CSI condition. However, the improvement is limited and is at the cost of higher complexity. We use this method for its low complexity and ease of analysis. The $l$ th component of vector $\mathbf{x}$ and the $l$ th row of matrix $\mathbf{x}$ are denoted by $\mathbf{x}(l)$ and $\mathbf{X}(l)$, respectively. The component-wise form is expressed as follows:

$$
\begin{aligned}
\mathbf{s}_{i+1}(l)= & \beta_{i}\left(\rho \hat{\mathbf{D}}_{i+1}(l)+k\left(\hat{\mathbf{E}}_{i+1}^{r}\right)^{\mathrm{H}} \overline{\mathbf{H}}_{i} \hat{\mathbf{E}}_{i}^{t}(l)\right) \mathbf{s}_{i} \\
& +k \beta_{i} \sum_{t \neq l}\left(\hat{\mathbf{E}}_{i+1}^{r}\right)^{\mathrm{H}} \overline{\mathbf{H}}_{i} \hat{\mathbf{E}}_{i}^{t}(t) \mathbf{s}_{i}+\left(\hat{\mathbf{E}}_{i+1}^{r}\right)^{\mathrm{H}} \mathbf{n}_{i+1}(l),
\end{aligned}
$$

where the received signal consists of three components: the information-carrying term, the interference term, and the noise term. The correlation matrix is approximate to the unit matrix. Therefore, the end-to-end channel capacity of $n$ phase outdated CSI system is changed as follows:

$$
C=M \log _{2}\left(1+\frac{1}{f(m)}\right)
$$

where 


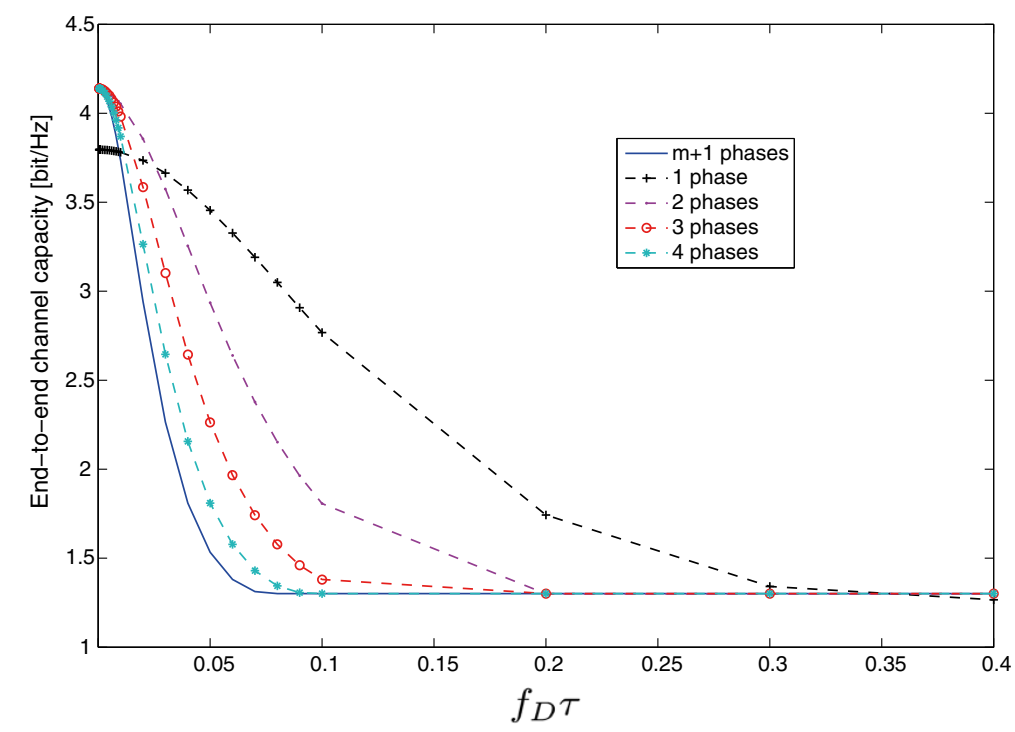

Figure 11 The end-to-end channel capacity of outdated CSI system that only has forward link interference. There were four relays.

$$
f(m) \approx \frac{\sigma^{2}+k(M-1) \sum_{i=0}^{m} l_{i, i+1} p_{i}+\sum_{i=1}^{m+1-n} l_{i-1, i+n} p_{i-1}+\sum_{i=0}^{m-1-n} l_{i}, i+1+n p_{i+1+n}}{\sum_{i=0}^{m} l_{i, i+1} p_{i}}
$$

for the system that has interference from both forward link and backward link.

$f(m) \approx \frac{\sigma^{2}+k(M-1) \sum_{i=0}^{m} l_{i, i+1} p_{i}+\sum_{i=1}^{m+1-n} l_{i-1, i+n} p_{i-1}}{\sum_{i=0}^{m} l_{i, i+1} p_{i}}$ for the system that has interference from only forward link (using MIMO beamforming).

Compared to the system that has perfect CSI at both the transmitter and the receiver (14), in the end-to-end channel capacity of the system that has outdated CSI at the transmitter (26), the co-channel interference by outdated CSI is added.

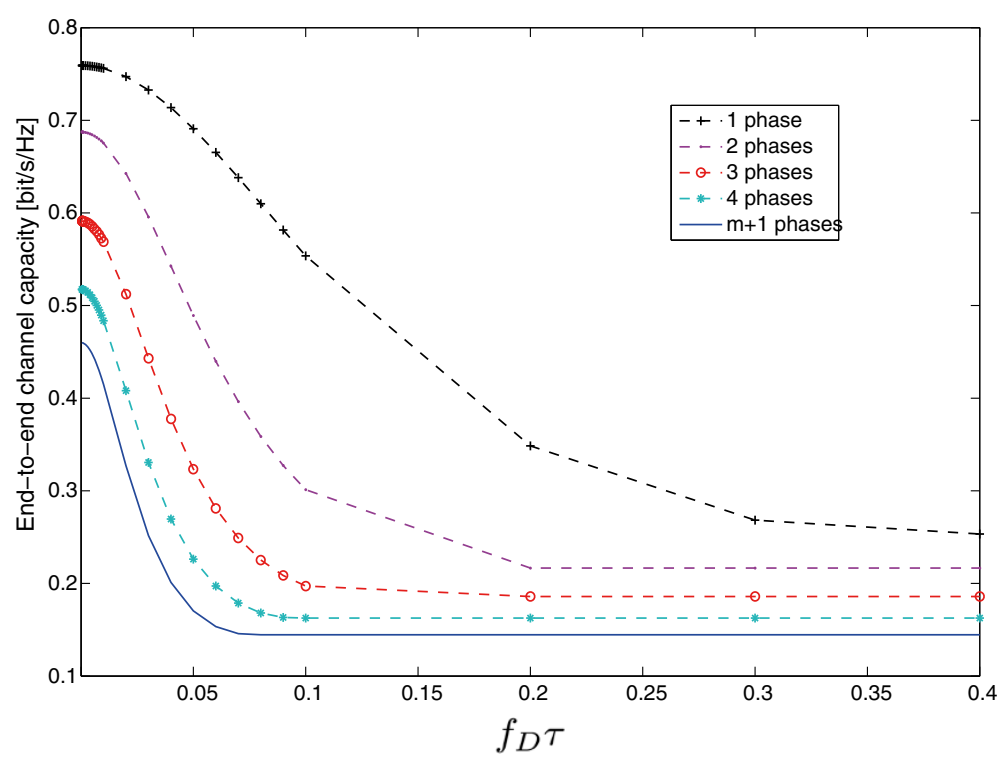

Figure 12 The end-to-end channel capacity of outdated CSI system in the case where transmission time is normalized. There were four relays. 


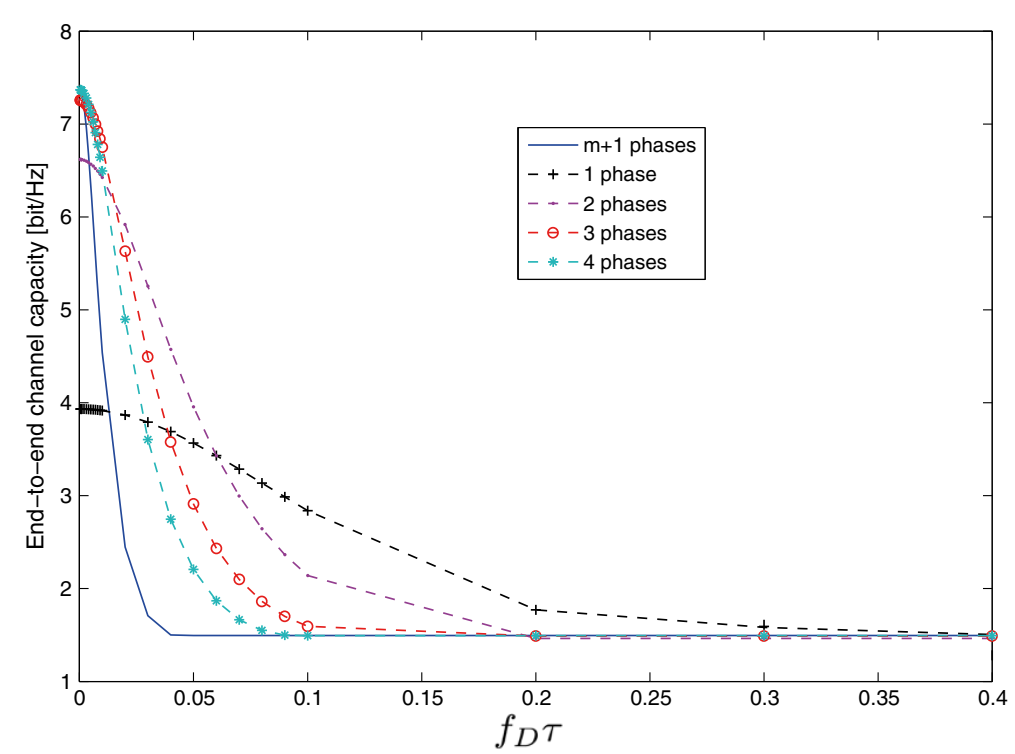

Figure 13 The end-to-end channel capacity of outdated CSI system that only has forward link interference. The were eight relays.

\subsection{End-to-end channel capacity of outdated CSI system}

The distance and the transmit power of the outdated CSI system is optimized by particle method (Subsection 3.2). The system model is the same as the one mentioned. The parameter is summarized in Table 1. As explained, the end-to-end channel capacity is changed depending on the number of relays. However, in this section, the end-toend channel capacity, depending on the outdated CSI, is examined. Therefore, we fix the number of relays.

Figures 11 and 12 show the end-to-end channel capacity of the system that only has the interference from the forward link (using the MIMO beam forming) where there are four numbers of relays. The end-to-end channel capacity of the system in the case where the transmission time is normalized is shown in Figure 12.

As shown in Figures 11 and 12, the end-to-end channel capacity is decreased when the term $f_{D} \tau$ increases. It can be explained that when the term $f_{D} \tau$ increases, the power of interference signal is increased; therefore, SINR decreases. The decrease of end-to-end channel capacity in each number of phases is different regardless of how large the end-to-end channel capacity is in the case of the perfect CSI system. The higher the number of phases, the larger the delay time becomes. Therefore, the end-to-end channel capacity of the system has the higher number of phases that decreased more rapidly. It means that the onephase transmission protocol is the most robust regardless of the number of relays. Figure 13 shows the end-to-end channel capacity of eight relay systems. Although the endto-end channel capacity of the one-phase system in the case of the perfect CSI (or when the term $f_{D} \tau$ is small) is lower than that of the higher number of phase systems, it becomes higher than the other higher number of phase systems when the term $f_{D} \tau$ increases.

\section{Conclusions}

In this paper, we analyzed the end-to-end channel capacity of multiple-hop MIMO relay system with MAC-PHY cross-layer. Compared to the system that has no control on the MAC layer, the end-to-end channel capacity of the system that has control on the MAC-PHY cross-layer is higher. It means that the trade-off of channel capacitycomplication is indicated. There is the optimum number of relays for each access control on the MAC layer that achieves the maximal end-to-end channel capacity. However, there is the trade-off between channel capacity and delay time. The system with outdated CSI is also analyzed, and the end-to-end channel capacity of the low number of phases is more robust than that of the higher number of phases regardless of the number of relays.

However, in this paper, the ergodic channel capacity has been analyzed, and we only proposed the MAC layer protocol; the appropriate modulation and code word were not considered. In the future, real-time channel capacity, the appropriate modulation, and code word will be examined. In addition, the optimal combination of the MAC layer and physical layer will be analyzed.

\section{Competing interests}

The authors declare that they have no competing interests.

\section{Author details}

${ }^{1}$ Division of Physics Electrical and Computer Engineering, Graduate School of Engineering, Yokohama National University, Yokohama, Kanagawa Prefecture 240-8501, Japan. 2Faculty of Radio-Electronics, Le Quy Don Technical University, 100 Hoang Quoc Viet, Cau Giay, Hanoi, Vietnam. 
Received: 17 December 2012 Accepted: 15 May 2013

Published: 29 May 2013

\section{References}

1. B Wang, J Zhang, A host-Madsen, On the capacity of MIMO relay channel. IEEE Trans. Inf. Theory. 51(1), 29-43 (2005)

2. DS Shiu, GJ Foschini, MJ Gans, JM Kahn, Fading correlation and its effect on the capacity of multi-element antenna systems. IEEE Trans. Commun. 48(3), 502-513 (2000)

3. D Gesbert, H Bolcskei, DA Gore, AJ Paulraj, MIMO wireless channel: capacity and performance prediction. Proc. GLOBECOM. 2, 1083-1088 (2000)

4. Y Liang, V Veeravalli, Gaussian Orthogonal relay channels: optimal resource allocation and capacity. IEEE Trans Inf. Theory. 51(9), 3284-3289 (2005)

5. K Lee, J Kim, G Caire, I Lee, Asymptotic ergodic capacity analysis for MIMO amplify-and-forward relay networks. IEEE Trans. Commun. 9, 2712-2717 (2010)

6. S Jin, M McKay, C Zhong, K Wong, Ergodic capacity analysis of amplify-and-forward MIMO dual-hop systems. IEEE Trans. Inf. Theory. 56(5), 2204-2224 (2010)

7. M Gastpar, M Vetterli, On the capacity of large Gaussian relay networks. IEEE Trans. Inf. Theory. 51(3), 765-779 (2005)

8. M Tsuruta, Y Karasawa, Multi-keyhole model for MIMO repeater system evaluation. IEICE Trans. Commun. J89-B(9), 1746-1754 (2006)

9. D Chizhik, GJ Foschini, MJ Gans, RA Valenzuela, Keyholes, correlations, and capacities of multi-element transmit and receive antennas. IEEE Trans. Wireless Commun. 1(2), 361-368 (2002)

10. G Levin, S Loyka, On the outage capacity distribution of correlated keyhole MIMO channels. IEEE Trans. Inf. Theory. 54(7), 3232-3245 (2010)

11. D Giindiiz, M Khojastepour, A Goldsmith, H Poor, Multi-hop MIMO relay networks: diversity-multiplexing trade off analysis. IEEE Trans. Wireless Commun. 9(5), 1738-1747 (2010)

12. P Razaghi, W Yu, Parity forwarding for multiple-relay networks. IEEE Trans. Inf. Theory. 55(1), 158-173 (2009)

13. P Hiep, R Kohno, Optimizing position of repeaters in distributed MIMO repeater system for large capacity. IEICE Trans. Commun. E93-B(12), 3616-3623 (2010)

14. PT Hiep, O Fumie, K Ryuji, Optimizing distance, transmit power and allocation time for reliable multi-hop relay system. EURASIP Journal on Wireless Communications and Networking. 2012, 153 (2012)

15. ITU-R, in Recommendation ITU-R P.526-8: propagation by diffraction. Radiowave Propagation (ITU-R Geneva, 2003)

16. ITU-R, in Recommendation ITU-R P.676-9: attenuation by atmospheric gases. Radiowave Propagation (ITU-R Geneva, 2001)

17. ITU-R, in Recommendation ITU-R P.838-2: Specific attenuation model for rain for use in prediction methods. Radiowave Propagation (ITU-R Geneva, 2001)

18. ITU-R, in Recommendation ITU-R P.1057-1: probability distributions relevant to radiowave propagation modelling. Radiowave Propagation (ITU-R Geneva, 2001)

19. ITU-R, in Recommendation ITU-R P.1238-3: propagation data and prediction methods for the planning of indoor radiocommunication systems and radio local area networks in the frequency range $900 \mathrm{MHz}$ to $100 \mathrm{GHz}$. Radiowave Propagation (ITU-R Geneva, 2003)

20. ITU-R, in Recommendation ITU-R P.1411-2: propagation data and prediction methods for the planning of short-range outdoor radiocommunication systems and radio local area networks in the frequency range $300 \mathrm{MHz}$ to 100 GHz. Radiowave Propagation (ITU-R Geneva, 2003)

21. N Kita, W Yamada, S Akio, Path loss prediction model for the over-rooftop propagation environment of microwave band in suburban areas. (in Japanese) IEICE Trans.Commun, J89-B(2), 115-125 (2006)

doi:10.1186/1687-1499-2013-144

Cite this article as: Thanh Hiep et al:: End-to-end channel capacity of MAC-PHY cross-layer multiple-hop MIMO relay system with outdated CSI. EURASIP Journal on Wireless Communications and Networking 2013 2013:144.

\section{Submit your manuscript to a SpringerOpen ${ }^{\mathcal{O}}$ journal and benefit from:}

- Convenient online submission

- Rigorous peer review

- Immediate publication on acceptance

- Open access: articles freely available online

- High visibility within the field

- Retaining the copyright to your article

Submit your next manuscript at $\boldsymbol{\nabla}$ springeropen.com 